\title{
Single-stage total hip arthroplasty after failed fixation of proximal femoral fractures: an increased risk for periprosthetic joint infections?
}

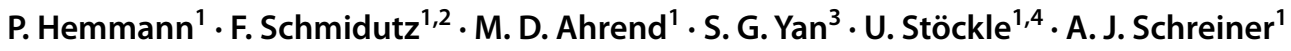

Received: 30 November 2020 / Accepted: 9 August 2021 / Published online: 28 August 2021

(c) The Author(s) 2021

\begin{abstract}
Background Higher complication rates have been reported for total hip arthroplasty (THA) after osteosynthesis of proximal femur fractures (PFF). This study evaluated the infection risk for conversion of internal fixation of PFF to THA by a singlestaged procedure in the absence of clear infection signs.

Methods Patients undergoing a one-staged conversion to THA (2013-2018) after prior internal fixation of the proximal femur were included. Preoperative diagnostics with laboratory results, hip aspirations as well as intraoperative microbiology and sonication were assessed. Postoperative complications were recorded as well as patient demographics, duration between initial and conversion to THA, explanted osteosynthesis and implanted THA.

Results Fifty-eight patients ( 24 male/34 female, $62.8 \pm 14.5$ years) were included with a mean time of $3.8 \pm 7.5$ years between internal fixation and conversion to THA ( 45 cementless, 3 cemented, 3 hybrid and 7 hybrid inverse THAs). Preoperative mean blood level CRP was $8.36 \pm 14 \mathrm{mg} / \mathrm{l}$ (reference value $<5 \mathrm{mg} / \mathrm{l}$ ) and leukocyte count was $7.11 \pm 1.84^{\wedge} 3 / \mu \mathrm{l}\left(4.5-10.000^{\wedge} 3 /\right.$ $\mu \mathrm{l})$. Fifty patients had intraoperative microbiological diagnostics, with either swabs in $86.2 \%$ and/or sonication in $29.3 \%$. Positive microbiological results were recorded in $10 \%$ (5 of 50 patients), with pathogens identified being mainly Staphylococcus. Complications after conversion occurred in $9.6 \%$ including a postoperative low-grade infection rate of $5.8 \%$ after a mean of 2.5 years.

Conclusion This study found a positive microbiological test result in $10 \%$ of a one-stage conversion of PFF fixation to THA. Moreover, we found a high infection rate (5.8\%) for early postoperative periprosthetic joint infection. Interestingly, CRP has not been proven to be an adequate parameter for low-grade infections or occult colonized implants. Therefore, we recommend a comprehensive pre- and intraoperative diagnostic including hip aspiration, swabs and sonication when considering one-staged revision.
\end{abstract}

Keywords Infection $\cdot$ PJI $\cdot$ Femoral fracture $\cdot$ Diagnostics

P. Hemmann and F. Schmidutz contributed equally to this work.

A. J. Schreiner

aschreiner@bgu-tuebingen.de

1 Department of Traumatology and Reconstructive Surgery, BG Trauma Center Tübingen, Eberhard Karls University Tübingen, Schnarrenbergstrasse 95, 72076 Tübingen, Germany

2 Department of Orthopaedic Surgery, Physical Medicine and Rehabilitation, University of Munich (LMU), Munich, Germany

3 Department of Orthopaedic Surgery, The First Affiliated Hospital of Anhui Medical University, Hefei, China

4 Center for Musculoskeletal Surgery (CMSC), Charité University Hospital Berlin, Berlin, Germany

\section{Introduction}

Proximal femoral fractures are one of the most common fractures with an incidence between 150 and 250/100,000 each year in industrial nations [1]. These fractures predominantly occur in elderly aged of more than 65 years (90\%) [2] and the incidence will further increase due to epidemiological development [3-5] with an expected doubling over the next 25 years [6].

Treatment usually includes internal fixation e.g., dynamic hip screw or intramedullary nails to preserve the natural hip joint. Nevertheless, high failure rates of up to $45 \%$ have been reported [6-9] and are related to femoral head necrosis, pseudarthrosis, cutting-out, peri-implant fractures as well as infection [10-12]. 
Revision usually results in conversion to total hip arthroplasty (THA). Unfortunately, studies reported clearly higher complication rates compared to primary THA [13, 14], including higher rates of dislocations, loosening and periprosthetic joint infection (PJI) $[15,16]$.

Periprosthetic infections are a devastating complication in elderly patients and might be referred to the multimorbid patient collective but also to previous surgery with orthopedic implants inserted. These implants are prone to occult infections [17] and are a result of bacteria adhesion and biofilm formation at the implant surface. Up to $50 \%$ occult infection rates of orthopedic implants have been reported [18] and can preoperatively not reliably be excluded with clinical and laboratory analysis, resulting in an increased risk for PJI [17, 19, 20].

Up to now, only few studies, most with small collectives, reported the outcome following surgical revision to THA. Thus, there is currently no international consensus, whether conversion of failed fixation to THA should be performed as a one- or two-stage procedure to reduce the risk of PJI. A single-stage procedure offers the advantage of only one surgical procedure and rapid mobilization on these elderly patients, while it potentially increases the risk of PJI.

The aim of this study was to evaluate the risk of PJI in one-staged conversion from failed fixation of PFF to THA in the absence of clinical infection signs.

\section{Materials and methods}

The hospital database was retrospectively (June 2013 to June 2018) screened for patients undergoing a one-staged conversion to THA after prior osteosynthesis of the proximal femur.

Patients were included with a history of a proximal femoral fracture (femoral neck fracture, trochanteric fracture) with initial osteosynthesis (dynamic hip screw, screws, intramedullary nail, plate) following a one-staged conversion to THA (cemented, cementless, hybrid, inverse hybrid THA).

Exclusion criteria were defined as no implantation of THA due to diagnosed or obvious signs of peri-implant or soft tissue infection.

The following parameters were retrospectively collected based on: gender, age at conversion, date of initial surgery, date of conversion surgery, duration between those two surgeries, preoperative blood sample levels of CRP and leukocytes, explanted osteosynthesis material (screws, dynamic hips screw, intramedullary nail, screws, plate) and the type of implanted THA (cemented, cementless, hybrid, inverse hybrid THA). Moreover, if available, results of preoperative diagnostic hip aspirations (alphadefensin, leukocytes, percentage of polymorphic cells) as well as intraoperative microbiology and sonication were collected and evaluated. However, preoperative diagnostic hip aspiration and intraoperative sonication were not used routinely in our hospital at that time.

The records were further screened for postoperative complications defined as soft tissue infection, PJI or revision surgery after THA. The follow-up was performed on our out-patient clinic.

Patient demographics and clinical parameters are presented descriptively as mean \pm standard deviation (minimum-maximum).

Receiver-operating characteristic (ROC) was calculated to analyze the test performance of preoperative CRP levels as a predictor for positive intraoperative swabs or PJI. We determined $p \leq 0.05$ as significant. Statistical analysis was performed with Microsoft $\odot$ Office Excel 2016 (Microsoft Corporation, Redmond, USA) and with JMP ${ }^{\circledR}$ (SAS Institute Inc., version 14.2, Cary, NC, USA). The study protocol was approved by the local ethics committee $(803 / 2018 \mathrm{BO} 2)$ of the university.

\section{Results}

\section{Patient collective}

After screening the records, a total of 58 patients ( 24 men, 34 women) were eligible for inclusion. The mean age at conversion surgery was $62.8 \pm 14.5$ years (19.0-91.3). The mean time between initial surgery and revision was $3.8 \pm 7.5$ years $(0-30.6)$. Early revision ( $\leq 6$ months) was necessary in 15 patients $(26 \%)$. The type of internal fixation removed included 17 screws, 26 dynamic hip screws, 7 intramedullary nails and 8 plates. Conversion to THA included 45 cementless THAs, 3 cemented THAs, 3 hybrid and 7 hybrid inverse THAs (Table 1), whereas revision components were used in 8 cases (13.8\%). 4 patients received revision stems, while 1 patient required a Burch-Schneider reinforcement cage and 1 patient needed a trabecular metal cup-cage construct. 2 patients received revisions stems and tripolar cups.

\section{Preoperative laboratory}

The preoperative laboratory examination showed a mean CRP value of $8.36 \pm 14 \mathrm{mg} / \mathrm{l}(0.1-87)$ (reference value $<5 \mathrm{mg} / \mathrm{l}$ ) and a mean leukocyte count of $7.11 \pm 1.84^{\wedge} 3 / \mu 1(3.3-11.2)$ (reference value $4.5-10.000^{\wedge} 3 /$ $\mu \mathrm{l}) .23$ patients presented an elevated CRP and 5 patients an elevated leukocyte count. 
Table 1 Demographic and clinical data (mean \pm standard deviation (minimummaximum) or $\mathrm{n}(\%)$ ) of the cohort

\begin{tabular}{|c|c|}
\hline \multicolumn{2}{|l|}{ Demographics of the cohort $(n=58)$} \\
\hline Age at revision & $62.8 \pm 14.5$ years $(19.0-91.3)$ \\
\hline Gender & $24(41 \%)$ male; $34(59 \%)$ female \\
\hline \multicolumn{2}{|l|}{ Data regarding surgeries } \\
\hline Duration first surgery and THA implantation & $3.8 \pm 7.5$ years $(0-30.6)$ \\
\hline \multirow[t]{4}{*}{ Explanted osteosynthesis } & $17(29 \%)$ screws \\
\hline & 26 (45\%) dynamic hip screws \\
\hline & $7(12 \%)$ intramedullary nails \\
\hline & $8(14 \%)$ plates \\
\hline \multirow[t]{4}{*}{ Implanted THA } & $45(78 \%)$ cementless THA \\
\hline & $3(5 \%)$ cemented THA \\
\hline & 3 (5) hybrid THA \\
\hline & $7(12 \%)$ inverse hybrid THA \\
\hline \multicolumn{2}{|l|}{ Data regarding infection and its diagnostic } \\
\hline Preoperative CRP & $8.36 \pm 14 \mathrm{mg} / \mathrm{l}(0.1-87)$ \\
\hline Preoperative leucocytes count & $7.11 \pm 1.84^{\wedge} 3 / \mu \mathrm{l}(3.3-11.2)$ \\
\hline Number of available intraoperative microbiological swabs & $50(86.2 \%$ of the total cohort) \\
\hline Number of available intraoperative sonication & $17(29.3 \%$ of the total cohort $)$ \\
\hline Intraoperative positive cultures & $\begin{array}{l}5 \text { ( } 10 \% \text { of available swabs, } 1 \text { swab } \\
\text { pos. with } 2 \text { pathogens) }\end{array}$ \\
\hline \multirow[t]{4}{*}{ Intraoperative detected pathogens } & $1 S$. aureus \\
\hline & 3 S. epidermidis \\
\hline & $1 S$. warneri \\
\hline & $1 S$. saccharolyticus \\
\hline \multirow[t]{4}{*}{ Follow-up cohort $(n=52)$ and observed complications } & $5(9.6 \%)$ overall complication rate \\
\hline & $3(5.8 \%)$ infection \\
\hline & $1(1.9 \%)$ aseptic cup loosening \\
\hline & $1(1.9 \%)$ aseptic stem loosening \\
\hline
\end{tabular}

\section{Intraoperative microbiology and sonication}

Microbiological swabs were available in $86.2 \%$ (50 of 58 patients) and implant sonication in $29.3 \%$ (17 of 58 patients), with $29.3 \%$ (17 of 58 patients) having microbiology and sonication. Overall, a positive culture was found in five patients $(10 \%)$.

In two patients, the microbiological swabs were positive whereby sonication was negative in one case and not performed in the other. Pathogens identified were $S$. aureus $(n=1)$ and $S$. epidermidis $(n=1)$.

In three patients, the microbiological swabs and sonication were positive. Identified pathogens were $S$. warneri $(n=1)$, S. epidermidis $(n=2)$ and S. saccharolyticus $(n=1)$, with one patient showing two pathogens (S. saccharolyticus, S. epidermidis).

\section{Preoperatively diagnostic hip aspiration}

In 14 cases (24.1\%), a preoperatively diagnostic hip aspiration was performed with one case being a sicca aspiration.
From the aspiration, microbiological cultures were examined in $92.8 \%$ (13 of 14 patients) with detection of S. epidermidis in one culture.

Alpha-defensin was also evaluated in these patients $92.8 \%$ (13 of 14 patients) but did not reveal a positive result: $0.1-0.2$ ( $>1.1$ high risk for infection).

Leukocyte cell count and percentage of polymorph cells could be analyzed in 57\% ( 8 of 14 patients), with 5 patients showing a cell count $<1000 / \mu \mathrm{l}, 1$ patient $1000-3000 / \mu \mathrm{l}$ and 2 patients $>3000 / \mu \mathrm{l}$. The polymorph cells showed a range of $19-75 \%$ with a mean percentage of $49.5 \%$

\section{Postoperative therapy}

All patients received an antibiotic prophylaxis intraoperatively, after taking the microbiological swabs. Antibiotic therapy was postoperatively continued until the final results were available after 14 days. In case of positive microbiological examination, patients received 6 weeks of combination therapy including two antibiotics to which the bacteria were susceptible. 


\section{Follow-up}

During the follow-up, two patients died who were not associated with conversion surgery. Another three patients were lost to follow-up due to lack of presentation. One patient moved abroad. The mean follow-up time of the remaining patients $(n=52)$ was 2.5 years $(0.5-5.6)$. Complications occurred in $9.6 \%$ of the patients, with one aseptic cup loosening (1.9\%), one aseptic stem loosening (1.9\%) and three low-grade infections (5.8\%). From these patients, none of them had a positive pre- or intraoperative microbiological result.

In eight patients, neither microbiological swabs nor sonication was available. One of the three low-grade infections was diagnosed within this group during follow-up. All other complications which were listed above were detected in the microbiologically tested group. Receiver-operating characteristics (ROC) curve for preoperative CRP as predictor for intraoperative positive microbiological swab or PJI was not significant ( $p=0.3793$ ).CRP (AUC: 0.3775$)$ demonstrated to be a poor fit (Fig. 1). An example of a patient requiring conversion surgery from internal fixation to THA is given in Fig. 2.

\section{Discussion}

This study evaluated whether a revision from internal fixation of proximal femoral fractures to THA can safely be performed as a one-staged procedure. Our results show a

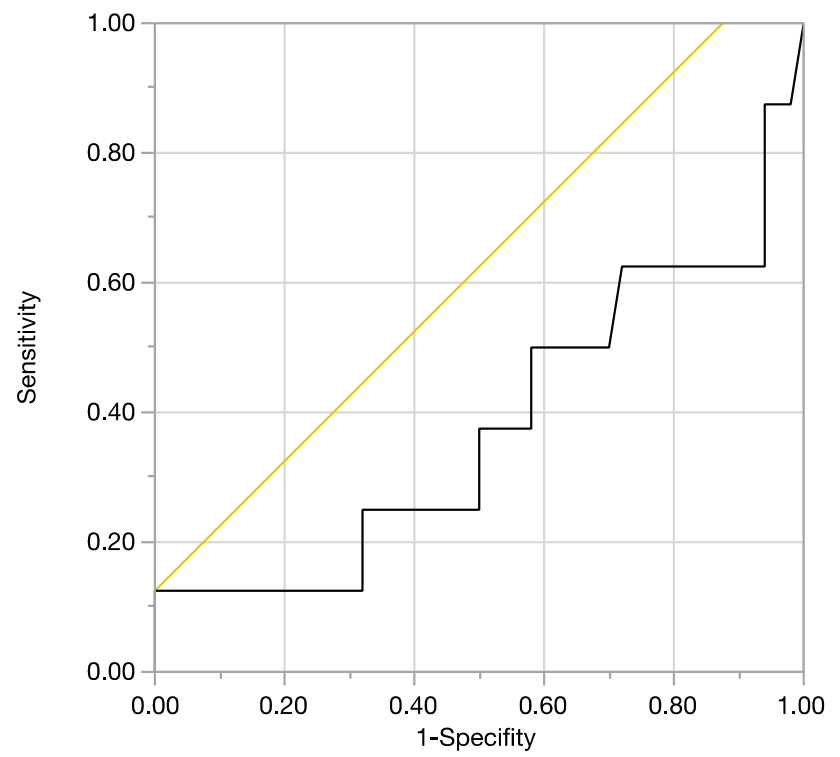

Fig. 1 Receiver-operating characteristics (ROC) curve for preoperative CRP as predictor for intraoperative positive microbiological swab or PJI. CRP was not a good predictor for an intraoperatively positive swab or later infection relevant number of intraoperative positive microbiological swabs and also higher revision rate compared to primary THA.

Positive microbiological results were found in $10 \%$ of the patients, despite the preoperative lack of clinical infection signs. Comparable to our results, Gittings et al. evaluated the conversion in 33 patients from internal femoral fixation to THA and found a high infection rate of $18 \%$ [21]. Kempthorne et al. found a rate of $15 \%$ positive cultures in patients with aseptic loosening for explanted THA or TKA implant $(n=54)$ [19]. These rates of occult infection are similarly reported in several studies evaluation removal of inconspicuous orthopedic implants [22, 23]. Knabl et al. evaluated osteosynthesis implants during routine removal after long bone fracture. The removed implants were assessed by sonication and PCR and the authors reported a positive microbiological culture in 56\% [24]. Another study by George et al. showed a positive culture rate of $51 \%$ in the explanted implants before THA [25]. In accordance to our and other studies, all patients had no clinical signs for infection prior implant removal [24]. The lower rate of positive results in our study compared to other studies $[21,24]$ is probably related to the different evaluation methods used, with PCR and sonication showing higher detection rates. Taking only our sonication results into account ( 3 out of 17), the positive rate also increases to $17.6 \%(n=3)$ in our collective.

The importance of sonification is strengthened by results of aseptic revisions in joint arthroplasty. Trampuz et al. documented a rate of $9 \%$ positive sonication cultures in a series of 54 removed hip and knee implants [26]. Rothenberg et al. reported for assumed aseptic revisions in THA and TKA a positive sonication rate of $15 \%$ [27]. Some studies certify sonication a higher sensitivity for microbiological diagnostic than the conventional methods $[17,28]$, but the higher rate of false-positive results has also to be noted [29, 30]. Nevertheless, sonication seems to be an essential part to improve detection and treatment of occult infection [28].

In our study, Staphylococcus (S. epidermidis, $n=3$ ), $S$. aureus, $S$. saccharolyticus and $S$. warneri $(n=1)$ were the predominant microorganism detected which is in accordance with previous studies describing coagulase-negative Staphylococcus as the most prevalent organism on osteosynthesis and arthroplasty devices [19, 20, 24, 27]. Coagulase-negative Staphylococcus are well known to produce a protective layer of biofilm [20] which does not induce acute inflammatory response, but results in low-grade infections $[23,26]$. Sonication is able to destroy the protective biofilm and allows the detection of those bacteria in the microbiological culture [23]. As a consequence, all patients with an intraoperative positive microbiological swab in our study were treated successfully with a calculated double antibiotic 
Fig. 2 49-year-old woman with failed screw osteosynthesis right 9 months after femoral neck fracture. THA 6 weeks after conversion surgery

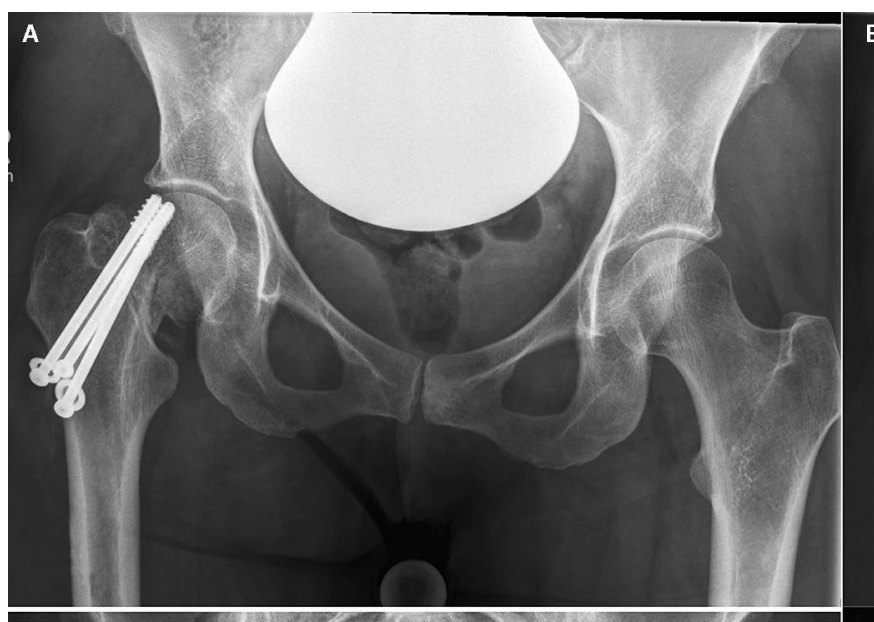

B
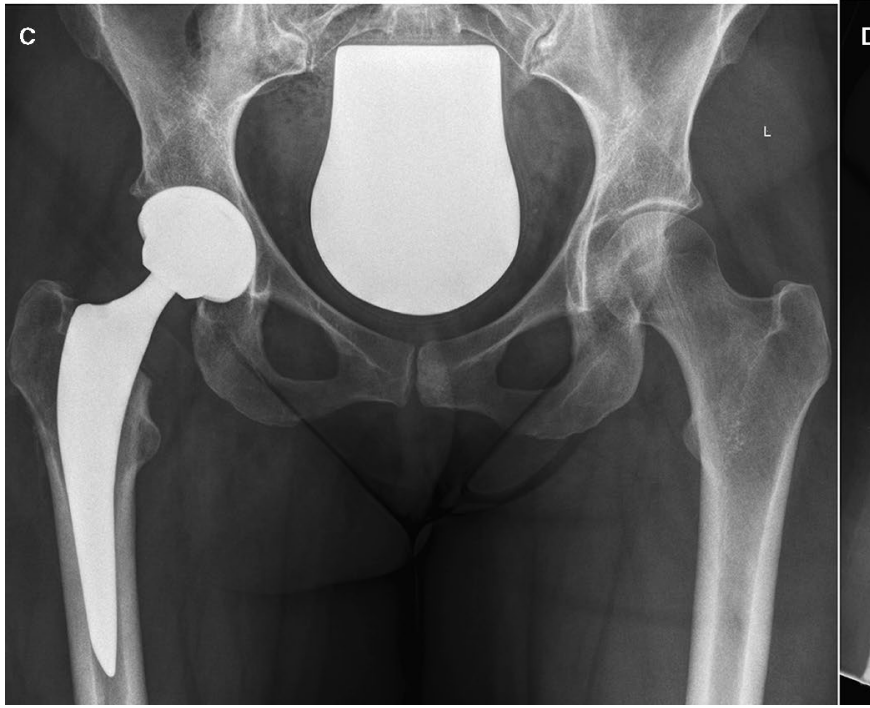

therapy for at least 6 weeks, according to an early PJI of THA.

The high colonization rate of osteosynthesis devices in clinical healthy patients is nowadays well known and a trigger for PJI [24]. However, it is not always clear whether implant failure (e.g., loosening) is caused by low-grade infection or infection is supported in the environment of loosening [19]. Still in all patients undergoing conversation to THA, an occult infection caused by a primary internal fixation must be considered [31] although detection is still challenging [32].

A preoperative laboratory with white cell blood count (WBC) and ESR or CRP are recommended but are nonspecific markers for PJI for low-grade infections in arthroplasty [33]. Gittings et al. showed that these markers are useful tools although occult infection could be missed [21]. They propose that patients with borderline or elevated inflammatory markers should be highly suspicious for infection. In our cohort, four out of five patients who had intraoperative positive microbiological swabs showed non elevated CRP blood levels postoperatively and 1 patient demonstrated a slightly elevated CRP value. In our cohort, the CRP blood level was not a good predictor for detecting occult colonized implants or low-grade PJI as seen by the ROC curve performed in this study. Pérez-Pietro et al. could also show that blood inflammatory markers like CRP level, or ESR may not be accurate enough especially when identifying low-grade and chronic PJI [34]. Therefore, aspiration with several tests such as, cell count, alpha-Defensin and microbiology seems to be a helpful tool for prevention of a PJI [35, 36]. If preoperative a PJI is suspected, we nowadays prefer a two-staged revision, although other authors still recommend a one-staged revision with antibiotics $[37,38]$ in favor of a single operation and a faster mobilization.

A complication rate of $9.6 \%$ was recorded during our postoperative follow-up. This rate appears to be high for THA, especially considering an early PJI rate of $5.8 \%$. Nevertheless, the data are in line with the current literature, and several studies even reported clearly higher numbers with a PJI risk ranging from 1.3 to $18 \%$ [13, 21, 39-41]. Several reasons might influence the increased infection risk and include the prolonged operation time [42], scar tissue 
in the approach for hip exposure, synovitis and hip stiffness and higher risk for intraoperative fractures due to osteopenia [16]. However, the PJI seems to be the highest risk due to the occult infection of the osteosynthesis devices. All patients diagnosed with a positive microbiology result were successfully treated with a combination therapy of two antibiotics for 6 weeks.

As a consequence of this retrospective study documenting the number of detected occult infections, we adopted our pre- and perioperative regime: all patients now receive: (1) preoperative laboratory incl. CRP and white cell count; (2) preoperative aspiration including a-Defensin, cell count with polymorph cells, CRP aspiration, Leucocyte Esterase test and microbiology/culture from the aspirate and (3) perioperative microbiological swabs/probes and sonication of the removed implants should follow to diagnose PJI. If preoperatively an (occult) infection is suspected according to the latest criteria of Parvizi et al. [43], a two-stage revision is performed. If one of the perioperative cultures is positive, we continue the combination therapy including two antibiotics for 6 weeks.

This study has several limitations which were connected to the retrospective study design. First, the study only analyzed patients with a single-stage revision and no comparative group with two-stage revision or primary THA was available. Second, the mean follow-up was relatively short and the evaluated data were presented descriptively in a heterogenous cohort. Third, the increasing knowledge in diagnostic PJI has clearly changed the concept of preoperative diagnostics which will clearly alter the outcome. Fourth, the postoperative infection rate might be underestimated since only 52 patients were evaluated for follow-up which implies a probable higher complication and infection rate than described. Nevertheless, it should be mentioned that nearly all complications occurred within the microbiologically tested group (which can, therefore, be considered a reliable testing tool).

\section{Conclusion}

Overall, we intraoperatively found an increased rate of positive microbiological results (10\%) during a one-staged revision when converting internal fixation of proximal femur fractures. We also found an increased risk for a PJI (5.8\%) which is in line with the current literature. However, in contrast to the increase PJI risk, the one-staged revision offers a faster mobilization and only one surgical procedure, potentially reducing peri- and postoperative complications. Interestingly, CRP has not been proven to be an adequate parameter for low-grade infections or occult colonized implants. In consequence of the results of this study, we recommend extensive pre- and intraoperative diagnostics including hip aspiration, microbiological swabs and sonication and in case of preoperative pathogen detection consideration of a twostaged procedure.

Author contributions PH: study design, statistical data analysis and interpretation, drafting and writing the manuscript. FS: study design, statistical analysis and interpretation, revision of the manuscript. MDA: statistical analysis and interpretation, revision of the manuscript. SGY: data interpretation, correction of the manuscript. US: supervision of the project, revision of the manuscript. AJS: data analysis and interpretation, drafting and writing the manuscript.

Funding Open Access funding enabled and organized by Projekt DEAL.

\section{Declarations}

Conflict of interest All the authors declare that they have no conflict of interest.

Open Access This article is licensed under a Creative Commons Attribution 4.0 International License, which permits use, sharing, adaptation, distribution and reproduction in any medium or format, as long as you give appropriate credit to the original author(s) and the source, provide a link to the Creative Commons licence, and indicate if changes were made. The images or other third party material in this article are included in the article's Creative Commons licence, unless indicated otherwise in a credit line to the material. If material is not included in the article's Creative Commons licence and your intended use is not permitted by statutory regulation or exceeds the permitted use, you will need to obtain permission directly from the copyright holder. To view a copy of this licence, visit http://creativecommons.org/licenses/by/4.0/.

\section{References}

1. Kanis JA et al (2012) A systematic review of hip fracture incidence and probability of fracture worldwide. Osteoporos Int 23(9):2239-2256

2. Bundes, G.d. Diagnosedaten der Krankenhäuser ab 2000 (Fälle, Berechnungs- und Belegungstage, durchschnittliche Verweildauer). Gliederungsmerkmale: Jahre, Behandlungsort, Alter, Geschlecht, Verweildauer, ICD10-4-Steller. Available from: http://www.gbe-bund.de/oowa921-install/servlet/oowa/aw92/ WS0100/_XWD_FORMPROC.

3. Benzinger $P$ et al (2016) The impact of preventive measures on the burden of femoral fractures - a modelling approach to estimating the impact of fall prevention exercises and oral bisphosphonate treatment for the years 2014 and 2025. BMC Geriatr 16:75

4. Icks A et al (2008) Trend of hip fracture incidence in Germany 1995-2004: a population-based study. Osteoporos Int 19(8):1139-1145

5. Rapp K et al (2008) Hip fractures in institutionalized elderly people: incidence rates and excess mortality. J Bone Miner Res 23(11):1825-1831

6. DeHaan AM et al (2013) Salvage hip arthroplasty after failed fixation of proximal femur fractures. J Arthroplasty 28(5):855-859

7. Koyuncu $S$ et al (2015) Mechanical failures after fixation with proximal femoral nail and risk factors. Clin Interv Aging 10:1959-1965 
8. Lu-Yao GL et al (1994) Outcomes after displaced fractures of the femoral neck. A meta-analysis of one hundred and six published reports. J Bone Jt Surg Am 76(1):15-25

9. Vajanto I et al (1998) Complications after treatment of proximal femoral fractures. Ann Chir Gynaecol 87(1):49-52

10. Archibeck MJ et al (2013) Total hip arthroplasty after failed internal fixation of proximal femoral fractures. J Arthroplasty 28(1):168-171

11. Min BW, Kim SJ (2011) Avascular necrosis of the femoral head after osteosynthesis of femoral neck fracture. Orthopedics 34(5):349

12. D'Arrigo C et al (2010) Hip arthroplasty for failed treatment of proximal femoral fractures. Int Orthop 34(7):939-942

13. Khurana $\mathrm{S}$ et al (2015) Total hip arthroplasty for posttraumatic osteoarthritis of the hip fares worse than THA for primary osteoarthritis. Am J Orthop (Belle Mead NJ) 44(7):321-325

14. Schnaser E, Scarcella NR, Vallier HA (2014) Acetabular fractures converted to total hip arthroplasties in the elderly: how does function compare to primary total hip arthroplasty? J Orthop Trauma 28(12):694-699

15. Gjertsen JE et al (2007) Total hip replacement after femoral neck fractures in elderly patients : results of 8577 fractures reported to the Norwegian Arthroplasty Register. Acta Orthop 78(4):491-497

16 McKinley JC, Robinson CM (2002) Treatment of displaced intracapsular hip fractures with total hip arthroplasty: comparison of primary arthroplasty with early salvage arthroplasty after failed internal fixation. J Bone Jt Surg Am 84A(11):2010-2015

17. Janz V et al (2015) Improved identification of polymicrobial infection in total knee arthroplasty through sonicate fluid cultures. Arch Orthop Trauma Surg 135(10):1453-1457

18. Zimmerli W, Trampuz A, Ochsner PE (2004) Prosthetic-joint infections. N Engl J Med 351(16):1645-1654

19 Kempthorne JT et al (2015) Occult infection in aseptic joint loosening and the diagnostic role of implant sonication. Biomed Res Int 2015:946215

20. Nelson CL et al (2005) Is aseptic loosening truly aseptic? Clin Orthop Relat Res 437:25-30

21. Gittings DJ et al (2017) Diagnosing infection in patients undergoing conversion of prior internal fixation to total hip arthroplasty. $\mathbf{J}$ Arthroplasty 32(1):241-245

22 Renard $\mathrm{G}$ et al (2019) Periprosthetic joint infection in aseptic total hip arthroplasty revision. Int Orthop 44:735-741

23. Bereza PL et al (2013) Identification of silent prosthetic joint infection: preliminary report of a prospective controlled study. Int Orthop 37(10):2037-2043

24 Knabl L et al (2018) High percentage of microbial colonization of osteosynthesis material in clinically unremarkable patients. Microbiologyopen 8:e00658

25. George $\mathrm{R}$ et al (2019) Is it safe to do a single-stage implant exit and primary hip replacement? Clinical and microbiological profiling. Indian J Med Microbiol 37(4):531-535

26. Trampuz A et al (2007) Sonication of removed hip and knee prostheses for diagnosis of infection. N Engl J Med 357(7):654-663

27. Rothenberg AC et al (2017) Sonication of arthroplasty implants improves accuracy of periprosthetic joint infection cultures. Clin Orthop Relat Res 475(7):1827-1836
28. Yano MH et al (2014) Improved diagnosis of infection associated with osteosynthesis by use of sonication of fracture fixation implants. J Clin Microbiol 52(12):4176-4182

29. Liu $\mathrm{H}$ et al (2017) The application of sonication in diagnosis of periprosthetic joint infection. Eur J Clin Microbiol Infect Dis 36(1):1-9

30. Trampuz A et al (2006) Sonication of explanted prosthetic components in bags for diagnosis of prosthetic joint infection is associated with risk of contamination. J Clin Microbiol 44(2):628-631

31. Tetsunaga $\mathrm{T}$ et al (2017) Total hip arthroplasty after failed treatment of proximal femur fracture. Arch Orthop Trauma Surg 137(3):417-424

32. Krause PC, Braud JL, Whatley JM (2015) Total hip arthroplasty after previous fracture surgery. Orthop Clin N Am 46(2):193-213

33. Berbari E et al (2010) Inflammatory blood laboratory levels as markers of prosthetic joint infection: a systematic review and meta-analysis. J Bone Jt Surg Am 92(11):2102-2109

34. Perez-Prieto D et al (2017) C-reactive protein may misdiagnose prosthetic joint infections, particularly chronic and low-grade infections. Int Orthop 41(7):1315-1319

35. Frangiamore SJ et al (2016) alpha-defensin accuracy to diagnose periprosthetic joint infection-best available test? J Arthroplasty 31(2):456-460

36. Riccio $\mathrm{G}$ et al (2018) Qualitative alpha-defensin versus the main available tests for the diagnosis of periprosthetic joint infection: best predictor test? J Bone Jt Infect 3(3):156-164

37. Zahar A, Gehrke TA (2016) One-stage revision for infected total hip arthroplasty. Orthop Clin N Am 47(1):11-18

38. Gehrke T, Kendoff D (2012) Peri-prosthetic hip infections: in favour of one-stage. Hip Int 22(Suppl 8):S40-S45

39. Weiss RJ et al (2012) Salvage of failed trochanteric and subtrochanteric fractures using a distally fixed, modular, uncemented hip revision stem. Acta Orthop 83(5):488-492

40. Enocson A et al (2012) Hip arthroplasty after failed fixation of trochanteric and subtrochanteric fractures. Acta Orthop 83(5):493-498

41. Scholten R et al (2019) High incidence of early periprosthetic joint infection following total hip arthroplasty with concomitant or previous hardware removal. Arch Orthop Trauma Surg 139(8):1051-1056

42. Willis-Owen CA, Konyves A, Martin DK (2010) Factors affecting the incidence of infection in hip and knee replacement: an analysis of 5277 cases. J Bone Jt Surg Br 92(8):1128-1133

43 Parvizi J et al (2018) The 2018 definition of periprosthetic hip and knee infection: an evidence-based and validated criteria. J Arthroplasty 33(5):1309-1314.e2

Publisher's Note Springer Nature remains neutral with regard to jurisdictional claims in published maps and institutional affiliations. 